University of South Carolina

Scholar Commons

7-2011

\title{
Adherence to Accelerometer Protocols Among Women From Economically Disadvantaged Neighborhoods
}

\author{
Patricia A. Sharpe \\ University of South Carolina - Columbia, SHARPEP@mailbox.sc.edu \\ Sarah Wilcox \\ University of South Carolina - Columbia, swilcox@sc.edu \\ Laura J. Rooney \\ Donna Strong \\ Rosie Hopkins-Campbell
}

See next page for additional authors

Follow this and additional works at: https://scholarcommons.sc.edu/

sph_physical_activity_public_health_facpub

Part of the Public Health Commons

\section{Publication Info}

Published in Journal of Physical Activity and Health, Volume 8, Issue 5, 2011, pages 699-706.

Sharpe, P. A., Wilcox, S., Rooney, L. J., Strong, D., Hopkins-Campbell, R., Butel, J., ... Parra-Medina, D. (2011). Adherence to accelerometer protocols among women from economically disadvantaged neighborhoods. Journal of Physical Activity and Health, 8(5), 699-706.

(c) Journal of Physical Activity and Health, 2011, Human Kinetics

This Article is brought to you by the Physical Activity and Public Health at Scholar Commons. It has been accepted for inclusion in Faculty Publications by an authorized administrator of Scholar Commons. For more information, please contact digres@mailbox.sc.edu. 


\section{Author(s)}

Patricia A. Sharpe, Sarah Wilcox, Laura J. Rooney, Donna Strong, Rosie Hopkins-Campbell, Jean Butel, Barbara E. Ainsworth, and Deborah Parra-Medina 


\title{
Adherence to Accelerometer Protocols Among Women From Economically Disadvantaged Neighborhoods
}

\author{
Patricia A. Sharpe, Sara Wilcox, Laura J. Rooney, Donna Strong, \\ Rosie Hopkins-Campbell, Jean Butel, Barbara Ainsworth, and Deborah Parra-Medina
}

\begin{abstract}
Background: Objective measurement of physical activity with accelerometers is a challenging task in community-based intervention research. Challenges include distribution of and orientation to monitors, nonwear, incorrect placement, and loss of equipment. Data collection among participants from disadvantaged populations may be further hindered by factors such as transportation challenges, competing responsibilities, and cultural considerations. Methods: Research staff distributed accelerometers and provided an orientation that was tailored to the population group. General adherence strategies such as follow-up calls, daily diaries, verbal and written instructions, and incentives were accompanied by population-specific strategies such as assisting with transportation, reducing obstacles to wearing the accelerometer, tailoring the message to the participant population, and creating a nonjudgmental environment. Results: Sixty women asked to wear the Actigraph GT1M returned the accelerometer, and 57 of them provided sufficient data for analysis (at least 10 hours a day for a minimum of 4 days) resulting in $95 \%$ adherence to the protocol. Participants wore the accelerometers for an average of 5.98 days and 13.15 hours per day. Conclusions: The high accelerometer monitoring adherence among this group of economically disadvantaged women demonstrates that collection of high-quality, objective physical activity data from disadvantaged populations in field-based research is possible.
\end{abstract}

Keywords: measurement, physical activity, exercise, low-income

Objective measurement of physical activity is a challenging task in community-based intervention research. While there are limitations to both self-report and objective measures of physical activity, use of accelerometers to assess physical activity is increasing in frequency to evaluate interventions in community settings. Participant adherence with physical activity monitoring has not received much attention ${ }^{1}$ and reports on the feasibility of and adherence to activity monitoring protocols among research participants from economically disadvantaged settings is particularly sparse. Recent reports using 2003-2004 data from the National Health and Nutritional Examination Survey have reported adherence with accelerometer monitoring (ie, providing at least 10 hours per day of data for a minimum of 4 days) of $74 \%$ among 3691 healthy white, black, and Hispanic adults aged 18 and older ${ }^{2}$ and $68 \%$ among 7176 ambulatory participants

Sharpe, Strong, Hopkins-Campbell, and Butel are with the Prevention Research Center, University of South Carolina, Columbia, SC. Wilcox is with the Dept of Exercise Science, University of South Carolina, Columbia, SC. Rooney is with the Dept of Health Promotion, Education, and Behavior, University of South Carolina, Columbia, SC. Ainsworth is with the Exercise and Wellness Program, School of Nutrition and Health Promotion, Arizona State University, Phoenix, AZ. Parra-Medina is with the Institute for Health Promotion Research, Dept of Epidemiology and Biostatistics, University of Texas Health Science Center San Antonio, San Antonio, TX. aged 6 and older. ${ }^{3}$ Adherence was not reported for population subgroups. ${ }^{2}$

There are general challenges to the use of accelerometers in all populations, including monitor loss, noncompliance with monitor wearing, and incorrect placement of monitors. Disadvantaged populations face additional challenges that may further hinder data collection, including transportation challenges, competing responsibilities and time constraints, relatively less experience with technology and research, and cultural differences. ${ }^{4-8}$ Variation in accelerometer wear can be associated with forgetfulness, participant motivation and desire to comply, and circumstances that impede wear (eg, activities like swimming or institutional/worksite prohibitions of wear) or affect patterns of wear (eg, employment hours, shift work, and irregular sleeping patterns). ${ }^{9}$ Objectively measuring physical activity participation using accelerometers is a relatively expensive and time-consuming undertaking. Skipped days or not enough hours of accelerometer wear, improper use and loss of accelerometers, attrition, and nonreturn are costly in terms of both data quality and research budget.

The purpose of this report is to describe participant recruitment, strategies used to enhance participant adherence, and accelerometer adherence rates among women from economically disadvantaged urban census tracks who enrolled in a community-based randomized controlled trial for weight loss. Although we report baseline physical activity levels among the participants, the 
primary purpose of the report is to describe strategies that researchers and practitioners can apply to obtain data of high quality, while minimizing accelerometer loss among underserved women.

\section{Pilot Test Methods and Results}

\section{Methods}

Sixteen women (11 African American and 5 white) participated in a pilot test of the accelerometer protocol in June and July of 2008. The women wore the accelerometer and completed a daily diary for 7 days from waking until bedtime. For all women, the accelerometer was placed in a pouch which was attached to a belt. Attention focused on practical issues of daily wear, such as remembering to put on the accelerometer, placement of the belt and pouch, and participant comfort. The 7-day pretest provided valuable insights into the challenges to adherence which informed the development of strategies for the primary study.

\section{Results}

The belts and pouches tended to move around and the belts were to be too small for larger women. Some women perceived the accelerometers as "ugly" with dress clothes and inappropriate to wear to some events (such as church). Revisions to the accelerometer orientation protocol included teaching women how to wear the accelerometer underneath clothing, if preferred, so it was not visible. The protocol was flexible enough to allow participants to wear the accelerometer beyond the original 7-day period, if needed to obtain enough days, so the women did not feel pressured to wear it on a day that included church or special events. The accelerometer was set to capture data for a 2 -week period of time.

Enhancements to the fit of belts and pouches included sewing a safety pin to the back of each pouch to secure the accelerometer to clothing and providing elastic belt extensions and extra plastic buckles (available at fabric stores and or through Internet-based suppliers) to accommodate larger waist circumferences. This enhancement was necessary because the largest belt size available from the ActiGraph distributor did not fit some of the women. An alternative to using the accelerometer pouch is to attach the ActiGraph units directly to the belt without the use of the pouch, which may reduce the placement slippage observed with the pouches.

\section{Primary Study}

\section{Methods}

Setting and Participants. The Institutional Review Board at the University of South Carolina approved this community-based randomized controlled trial of a behavioral weight loss intervention.
Researchers recruited women from 18 urban contiguous census tracts in central South Carolina where $25 \%$ or more of the population had income below the poverty level. Recruitment methods included word of mouth, fliers posted in diverse community settings, posters on city buses, and presentations and information tables at community events and gatherings. Participant recruitment took place during September through November 2008, and measurement sessions occurred during November and December 2008.

Inclusion criteria for participation in the weight loss intervention included residency within identified census tracts, age between 25 and 50 years, able to speak and understand English, increased risk of chronic disease morbidity and mortality (waist circumference greater than $88 \mathrm{~cm}$ ), ability to participate in moderate physical activity and absence of physical or psychological impairment that would preclude participation in intervention activities. Zhu et $\mathrm{al}^{10}$ found that waist circumference better indicates cardiovascular disease risk across non-Hispanic black, non-Hispanic white, and Mexican Americans than does BMI, and identified $83.3 \mathrm{~cm}$ as the average waist circumference corresponding to BMIs equal to 25.0 for women of all races with 1 or more cardiovascular disease risk factors. The American College of Sports Medicine classifies women with waist circumferences greater than $88 \mathrm{~cm}$ who are overweight $(\mathrm{BMI}=25.0-29.9)$ or obese (BMI > 30.0) as having high to extremely high disease risk (Type 2 diabetes, hypertension, and cardiovascular disease). ${ }^{11}$ The inclusion criterion of waist circumference of at least $88 \mathrm{~cm}$ ensured participants recruited for the weight loss intervention were women for whom significant weight loss was warranted.

\section{Procedures}

Overview of the Measurement Session. The study's baseline measurement session included informed consent and orientation to study expectations; anthropometric measures and health history; the Physical Activity Readiness Questionnaire (PAR-Q), ${ }^{12}$ a psychosocial questionnaire; orientation to the accelerometer (ActiGraph model GT1M) and dietary recall protocols; and the first of 3 phone-based dietary recall interviews. During the informed consent process, research staff described the study requirements, including the wearing of an accelerometer (referred to as a motion sensor) for 7 days, at least 10 hours per day. After the informed consent process, research staff guided women through a series of measurement stations.

Orientation to the Accelerometer. At the accelerometer station, staff used a standard script to describe the accelerometer protocol in full detail. The accelerometer orientation was interactive; each woman was asked if she had questions about instructions along the way, scripted prompts were used to check for understanding, and each woman was invited to come up with examples of storage locations and memory aids she might use. 
Research staff showed the woman an accelerometer, belt, and pouch and explained that the motion sensor had an internal sensor that would keep track of her body movement throughout the day. To reduce suspicion, the staff person assured each woman that the motion sensor does not keep track of her location. Researchers described the purpose of the week's wear as "giving us an idea of what a typical week is like for you now." The staff person emphasized that wearing the accelerometer was voluntary and there was no judgment if she changed her mind about participating; also that the motion sensor was a valuable research device that needed to be returned to the project.

Each woman was fitted with a belt and pouch, shown the proper placement for wear, provided instructions for completing a simple daily diary about her daily activities (described below in more detail) and scheduled an appointment to return the accelerometer after 7 days. Throughout the accelerometer orientation session, research staff repeated the need for 7 days of at least 10 hours of wear time and the importance of returning the accelerometer. The research staff also stressed the importance of making no changes to her existing physical activities and that she should "just go about your normal activities."

All scripts and written materials used the term "motion sensor" rather than accelerometer to minimize the use of technical terms that may be unfamiliar to women. The researcher's script included instructions for storing the accelerometer while not in use (eg, out of the reach of small children and pets) and while sleeping (eg, place it where she would remember it each morning). At the end of the orientation, the researcher asked the woman to repeat the instructions and clarified any remaining misunderstanding. Each woman put her own accelerometer on to wear home to demonstrate how and where to place the monitor and to ensure proper fit of the elastic belt. The script is available online at http://prevention.sph.sc.edu/tools/.

Accelerometer Diary. Each woman was instructed on how to record basic information in the accelerometer diary, including the time the accelerometer was put on and taken off, periods of nonwear (eg, during showering, bathing, or swimming) and exercise times and activity types. The return appointment date and time were written on the accelerometer diary.

The accelerometer diary was adapted from the previous work of colleagues to suit current participants based on feedback received from pretesting. ${ }^{13}$ The first 2 pages of the diary contained a summary of instructions given during the orientation. The list of tips and reminders was written with the same terms used during the orientation and included a picture of proper accelerometer placement. Additional pages were included to record the details for each day of wear. The diary also included a page per day for 7 days on which to record the following: today's date; the time the motion sensor was put on; whether the sensor was taken off for more than 20 minutes during the day (yes/no); whether the participant exercised that day (yes/no) and if so, start time, stop time, and type of exercise; and the time the motion sensor was taken off. The term "exercise" was used in the diary to refer to planned activity, as opposed to "physical activity" based on our previous research with women, which indicated a preference for the term "exercise" to describe intentional activity. ${ }^{14}$ The diary was prepared at a Flesch-Kincaid reading level of 6.3, and its user-friendly format featured fill-in boxes, short questions, and response choices that could be circled. An example of the diary can be seen online at http://prevention.sph.sc.edu/tools/.

Measurement Session Check-Out. At the end of the measurement session, instructions were reviewed and the accelerometer return appointment reconfirmed. Participants were reminded a final time of the need for 7 days of at least 10 hours of wear time and the importance of returning the accelerometer. Each woman received a pocket folder with the research coordinator's direct phone number and an alternate number written on the inside pocket. She also received an appointment slip for returning the monitor and was told that she would receive 3 reminder telephone calls about wearing the accelerometer and could ask any additional questions at those times. Each woman was told that if she decided not to participate after leaving, she should call the project office so that research staff could arrange to get the motion sensor back. Participants received $\$ 35$ for the baseline measurement session and were informed that they would receive an additional $\$ 45$ upon return of the accelerometer with usable data as previously explained. A tracking database was used to monitor accelerometer assignment (by ID number) for each participant, followup appointment dates and incentive disbursements.

Reminder Calls. Researchers confirmed contact information and collected preferred times for morning reminder calls during the upcoming week at the measurement session. During the following week, research staff using standard scripts called each woman 3 times to remind her to wear her accelerometer and to answer any questions. The staff called participants on the first day of accelerometer wear (the day after the orientation), the fourth day of wear and the seventh (last) day of wear. During the last call, research assistants reminded the participant of her scheduled appointment to return the accelerometer and activity diary to the project office. Research staff tracked communication with participants by noting the results of each phone call.

Protocol for Accelerometer Collection. When the participant returned the accelerometer, the research assistants downloaded and visually inspected the data to ensure that data were available on at least 4 days for at least 10 hours per day. If an adequate number of days was not observed, the participant had the opportunity to wear the accelerometer for additional days, return the monitor with sufficient data and receive the \$45 incentive at the later time. If participants with insufficient data 
agreed to wear the accelerometers longer, they received another activity diary.

\section{Data Analysis}

Descriptive statistics (means, standard deviations, and frequencies) were used to summarize all data, including sociodemographic, anthropometeric, and behavioral characteristics of participants at baseline; participant recruitment and screenings; telephone reminder calls; participant adherence to the protocol; accelerometer wear time; and time spent in moderate- and vigorous-intensity physical activity.

Daily accelerometer data were considered valid if the monitor was worn for at least 10 hours per day. Nonwear was assumed for any consecutive strings of 0 counts for 20 or more minutes. To avoid introducing systematic bias into the data of women who had more than 7 days of wear, the data were examined to identify which weekdays showed $\geq 10$ hours of counts. Only comparable substitutions were made (ie, the recorded (makeup) weekday's data were used in place of a comparable omitted weekday, such as recorded Tuesday in place of missed Tuesday), and only when necessary to obtain 4 days of data. For adults, 3 to 4 days of monitoring is required to achieve acceptable reliability estimates. ${ }^{15}$ Three days of monitoring has been the criterion used most often, ${ }^{16}$ and 3 to 5 days are recommended to estimate habitual physical activity. ${ }^{9}$ In the 2003-2004 National Health and Nutritional Examination Survey (NHANES), participants were included if they had 4 or more valid days of monitor wear. ${ }^{3}$ Ten hours per day is the most common cut-point used in accelerometer studies ${ }^{16}$ and was the cut-point used in NHANES. ${ }^{3}$

One-minute epochs are most commonly used in adult accelerometer studies ${ }^{1}$ and were used in this study. Intensity thresholds developed by Freedson, Melanson, and Sirard ${ }^{17}$ were used to determine moderate and vigorous physical activity levels. Thresholds for moderate intensity physical activity were counts per minute between 1952 and 5724 and vigorous intensity physical activity were counts per minute $\geq 5725$.

\section{Results}

\section{Recruitment and Participation}

Of 158 women screened for initial eligibility by phone, 85 women scheduled in-person eligibility determination and enrollment appointments. Two women were excluded, and 23 cancelled or missed their appointments. The remaining 60 women attended a baseline measurement session.

\section{Reminders}

Research assistants called each participant $(n=60) 3$ times during the week for a total of 180 reminder calls. Inperson confirmation of accelerometer wear was obtained for 111 of the calls (61.7\%), a voice message was left 52 times $(28.9 \%)$, and follow-up attempts with no contact at all occurred 17 times $(9.4 \%)$.

\section{Adherence to the Protocol}

Research staff distributed 60 accelerometers. None of the pilot study participants were included in this sample. Fifty-nine participants returned the accelerometers and diaries at follow-up appointments, and a researcher collected 1 accelerometer in person in the community, thus there was no loss of accelerometers. A majority $(n=54)$ of participants returned the accelerometers and completed the diaries during the initial 7-day period, with sufficient data according to visual review at the return appointment. Six women did not have sufficient data and agreed to wear the accelerometers for additional days and record their daily activities to obtain sufficient data. Of these, 3 participants did not obtain a total of 4 days with 10 hours of wear and were excluded from these analyses, thus the final adherence level was $95 \%$ (57 of 60). Table 1 reports the sociodemographic and anthropometric characteristics of the 57 women who provided sufficient data for analysis.

Diary return and completion rates were high. Fiftynine diaries were returned out of the 60 that were distributed (98.3\% return rate). Fifty-four diaries contained complete information about the time the motion sensor was put on and taken off $(91.5 \%)$; 47 women completed the question of whether they had exercised (79.7\%); and 43 women completed the question of whether they took off the motion sensor for more than 20 minutes $(72.9 \%)$ for all 7 days of monitoring. Only 5 diaries were returned with completely missing days or entire categories of information missing. Table 2 describes accelerometer wear and physical activity level, including mean number of days worn, mean wear time per day and minutes per day spent in moderate or vigorous intensity physical activity. Participants wore the accelerometers for a mean of 5.98 days (range $=4.00,12.00$ ) and approximately 13.15 hours per day (range $=8.77,21.95)$. Accelerometers recorded a median of 11.0 minutes (mean of 17.9 minutes) of moderate and less than 1 minute of vigorous physical activity during each day of wear. Twenty of the 57 women $(35 \%)$ had 1 or more days of wear that included at least 1 10-minute bout of moderate or vigorous intensity physical activity.

\section{Discussion}

The high rate of adherence $(95 \%)$ to the accelerometer protocol compares favorably with adult NHANES participants $(74 \%)^{2}$ and indicates that a group typically deemed "hard to reach," that of economically disadvantaged and minority women, can provide objectively monitored physical activity data of sufficient quality at a high rate of completion. Key to enhancing adherence to physical activity monitoring protocols is creating an adherence 
Table 1 Characteristics of Women Participating in Accelerometer Monitoring $(n=57)$

\begin{tabular}{|c|c|c|c|c|}
\hline & Mean & SD & Min & Max \\
\hline Age (years) & 37.2 & 8.0 & 25.0 & 49.0 \\
\hline Weight (lbs.) & 243.9 & 56.4 & 149.4 & 390.0 \\
\hline \multirow[t]{2}{*}{ Waist Circumference $(\mathrm{cm})$} & 119.3 & 16.2 & 88.0 & 150.3 \\
\hline & \multicolumn{2}{|c|}{ n } & \multicolumn{2}{|c|}{$\%$} \\
\hline \multicolumn{5}{|l|}{ Body Mass Index (BMI) } \\
\hline $25.0-29.9$ & \multicolumn{2}{|c|}{4} & \multicolumn{2}{|c|}{7.0} \\
\hline$\geq 30.0$ & \multicolumn{2}{|c|}{53} & \multicolumn{2}{|c|}{93.0} \\
\hline \multicolumn{5}{|l|}{ Race } \\
\hline Black/African American & \multicolumn{2}{|c|}{50} & \multicolumn{2}{|c|}{87.7} \\
\hline White & \multicolumn{2}{|c|}{5} & \multicolumn{2}{|c|}{8.8} \\
\hline$>1$ race & \multicolumn{2}{|c|}{2} & \multicolumn{2}{|c|}{3.5} \\
\hline \multicolumn{5}{|l|}{ Ethnicity } \\
\hline Hispanic & \multicolumn{2}{|c|}{4} & \multicolumn{2}{|c|}{7.0} \\
\hline Non-Hispanic & \multicolumn{2}{|c|}{53} & \multicolumn{2}{|c|}{93.0} \\
\hline \multicolumn{5}{|l|}{ Employment status } \\
\hline Employed or self-employed & \multicolumn{2}{|c|}{42} & \multicolumn{2}{|c|}{73.7} \\
\hline Not employed outside the home & \multicolumn{2}{|c|}{9} & \multicolumn{2}{|c|}{15.8} \\
\hline Student & \multicolumn{2}{|c|}{2} & \multicolumn{2}{|c|}{3.5} \\
\hline Unable to work & \multicolumn{2}{|c|}{4} & & \\
\hline Level of education & & & & \\
\hline Some high school & & & & \\
\hline High school graduate/GED & & & & \\
\hline Some college/technical school & & & & \\
\hline College graduate & & & & \\
\hline Marital status & & & & \\
\hline Married \& living w/ partner & & & & \\
\hline Divorced & & & & \\
\hline Married but separated & & & & \\
\hline Unmarried couple \& living together & & & & \\
\hline Never married & & & & \\
\hline Children $<18$ years in the home & & & & \\
\hline 0 children & & & & \\
\hline$\geq 1$ child & & & & \\
\hline Health care coverage & & & & \\
\hline Yes & & & & \\
\hline No & & & & \\
\hline
\end{tabular}

Note. This table reflects data from participants who wore the accelerometer and had usable data $(\mathrm{n}=57 \mathrm{of} 60)$.

Table 2 Objectively Measured Physical Activity of Women Participating in Accelerometer Monitoring $(n=57)$

\begin{tabular}{lccccc}
\hline & Mean & Median & SD & Min & Max \\
\hline Days of valid wear $^{\mathrm{a}}$ & 5.98 & 6.00 & 1.34 & 4.00 & 12.00 \\
Hours of wear per day & 13.15 & 13.00 & 2.05 & 8.77 & 21.95 \\
Minutes of moderate Intensity PA $^{\mathrm{b}}$ & 17.92 & 11.00 & 19.87 & 0.00 & 157.00 \\
Minutes of vigorous intensity PA $^{\mathrm{b}}$ & 0.12 & 0.00 & 1.43 & 0.00 & 25.00 \\
\hline
\end{tabular}

a A valid day was defined as $\geq 10$ hours of wear; nonwear was assumed for any consecutive strings of 0 counts for $\geq 20$ minutes.

${ }^{\mathrm{b}}$ Thresholds for moderate intensity PA were counts per minute between 1952 and 5724 and vigorous intensity PA were counts per minute $\geq 5725 .{ }^{17}$ 
enhancement strategy tailored to the study population. Trost et $\mathrm{al}^{1}$ recommended including a combination of investigator-based and participant-based strategies to promote adherence with protocols. Investigator-based strategies are activities that the researchers initiate (eg, follow-up calls to participants during the measurement period) to assist participants in following protocols successfully while participant-based strategies are additional tasks (eg, activity diaries) designed to promote adherence. In addition to employing general adherence strategies, considering the specific needs and challenges of the study population is also important..$^{5}$ The components of the adherence strategy (outlined in Table 3 ) incorporated investigator and participant-based activities as well as addressed challenges to adherence that may be unique to economically disadvantaged research participants.

Research staff took special care to make the participant comfortable during all interactions and to ensure that the woman understood all the instructions for wearing the accelerometer. Women comprised the entire 10-person

\section{Table 3 Strategies to Promote Adherence to Accelerometer Protocols}

\begin{tabular}{ll}
\hline Adherence strategy & Details \\
\hline $\begin{array}{l}\text { Accelerometer wear and care tips } \\
\text { and reminders }\end{array}$ & $\begin{array}{l}\text { Each participant received reminders and tips for wearing the accelerometer correctly } \\
\text { and caring for the accelerometer at home. }\end{array}$ \\
Reminder telephone calls & $\begin{array}{l}\text { Each participant received 3 follow-up telephone calls during the 7-day monitoring period } \\
\text { (1st day, 4th day, and 7th day). }\end{array}$
\end{tabular}

Physical activity diary

Diaries were easy-to-use to minimize participant burden by having answer boxes and prepared response options.

Identifying and overcoming

Pretest of accelerometer protocols and 7-day wear informed research staff about potential obstacles to wearing accelerometer obstacles to wearing the monitor during daily activities.

Incentives contingent on adherence Monetary incentives were contingent upon visual inspection of graphs of Actigraph data (ten hours of activity on at least 4 days).

\begin{tabular}{ll}
\hline Adherence strategy & Details \\
\hline $\begin{array}{l}\text { Identifying \& overcoming obsta- } \\
\text { cles to wearing accelerometer }\end{array}$ & $\begin{array}{l}\text { Based on pretest feedback, safety pins were sewn onto the back of each pouch to assist } \\
\text { with belts and pouches moving around on larger, shapely bodies and women were taught } \\
\text { how to wear the monitor under clothing so it was not "unsightly" with dress clothes. }\end{array}$
\end{tabular}

Transportation \& parking assistance for attending sessions

Tailored, interactive accelerometer orientation based on patient education principles

Compatibility of research staff

Participants viewed as partners in research

Nonjudgment
Participants received parking passes and incentives for attending measurement sessions to defray the expenses of participation.

Accelerometer orientation included repetition of key information, verbal and written instructions (which were written at low reading levels), demonstrations and illustrations, confirmation of understanding and trusting environment where participants were free to ask questions.

The entire 10-person research staff was female (5 Caucasian, 5 African American). Lead staff were trained in cultural competence. All staff were certified in research with human subjects.

Research staff engaged participants as partners in the data collection process by sharing information about the value of the accelerometers as a research tool, and acknowledging how each woman's contribution made a difference in advancing research and community programs.

Continuous reinforcement from research staff regarding the voluntary contributions of the participants and that no judgment regarding activity levels or adherence would take place. 
research team (5 white, 5 African American) to enhance participants' comfort with both accelerometer orientation (eg, fitting a belt) and other aspects of measurement (eg, waist circumference taken under clothing). The orientation session used basic strategies for enhancing adherence, including repetition of key information, reinforcement of verbal instructions with written instructions, use of demonstrations and illustrations, use of simple instructions, written materials of appropriate reading levels, and participants' repetition of instructions back to research staff to confirm understanding.

Throughout the orientation and follow-up calls, participants were encouraged to ask questions and reminded that there was no judgment made about their activity data or results and they always had permission to change their minds about participation. The honest disclosure of the monetary value of the accelerometer itself and the importance of the data being collected to the research project was stated in such a way as to make the woman a partner in the research process as opposed to conveying suspicion regarding theft or carelessness.

Allowing the women to keep the accelerometer longer than 7 consecutive days (up to 14 days), if needed, to make up for forgotten days may be critical for obtaining 3 to 5 days of usable data to evaluate the impact of behavioral interventions. Such allowances may be especially important when participants will be asked for repeated waves of data collection; however, care must be taken in data management and analysis to avoid introducing systematic bias. To avoid bias, (eg, the inclusion of all weekdays and no weekend days) while obtaining at least 4 usable days of data, our data were examined for which days of the week with $\geq 10$ hours were available. Only comparable substitutions were made (ie, the recorded (makeup) weekday count in place of the comparable omitted weekday) and only when necessary to obtain 4 days of data. There were in fact few women $(n=6)$ who initially had insufficient data and needed to keep the accelerometer longer than 7 days to make up of missed days or days with insufficient hours of wear. While imputation is an alternative approach, we preferred using actual data as long as comparable days of the week could be substituted for missing days.

The high accelerometer adherence $(95 \%)$ and return rates (zero loss) among this group of economically disadvantaged women demonstrates that collection of objective physical activity data from special populations in field-based research is possible. The lack of attention given to accelerometer adherence strategies in the research literature makes it difficult to compare the observations in this report with the outcomes of other research using accelerometry to measure physical activity. The strategies to enhance accelerometer adherence described in this report appeared successful. Applying adherence-promoting strategies described in Table 3, such as approaching the research participant as a partner, verbalizing a lack of judgment concerning activity level, and anticipating and addressing likely challenges the women would face were key ingredients. The provision of an adequate incentive payment may have had a particularly positive impact on return of the accelerometer and data quality. Financially disadvantaged women have transportation and childcare costs associated with coming to the university to participate in research that may create a significant burden. The provision of an incentive that is significant but not so large as to be coercive conveys respect for the participants' time and effort.

The use of an activity diary is not essential and adds somewhat to participant burden, however, though our diary data quality and rate of return were good. While the diary was in a simple pen-and-paper format for this group, young and technology-minded study participants may prefer electronic and interactive diary formats. The value of having participants record their minutes of physical activity and type of physical activity on each day of wear is the ability to compare the women's perceived time spent in moderate or vigorous physical activity to the accelerometer data for the day. In theory, women participating in a behavioral intervention would develop a better understanding of moderate and vigorousintensity and would therefore show greater agreement between their self-reports and the accelerometer counts for time spent in moderate or vigorous-intensity activities at posttest than at baseline. The diary also records the occurrence of moderate-intensity activities that may not be sufficiently captured with an accelerometer (eg, swimming, gardening).

While adherence to the accelerometer protocol was high, physical activity levels were low. Based on the Freedson et al cut-points of 1952 to 5724 counts per minute, the participants accumulated a median of 11.0 minutes per day (mean of $17.92 \pm 19.87$ minutes per day) in moderate-intensity physical activities, which is well below the recommendation of 30 minutes per day, ${ }^{18}$ but is similar to values reported by 20- to 59-year-old women in NHANES. ${ }^{3}$ In addition, these values reflect total minutes and not bouts of activity. There is speculation that these cut-points may be too high to reflect moderate-intensity by overweight and obese persons, ${ }^{19}$ who have slower walking pace and different gait patterns than persons of desirable weight. ${ }^{19,20}$ Additional studies are needed to examine the contribution of adherence to accelerometer protocols and the relevance of cut-point thresholds to understanding the effectiveness of physical activity interventions in overweight and obese, economically disadvantage populations.

This report used cross-sectional data from baseline measurement for an intervention study. To date, we do not have data on attrition for repeated measurement over time, the potential for a decline in adherence or degradation of data quality. Future studies using accelerometry to monitor physical activity should include adherence rates and reports of adherence enhancing strategies (successful and unsuccessful) as a part of standard reporting of measurement methods to facilitate the development of a set of best practice procedures and provide attention to the adherence component of the physical activity monitoring literature. Additional reports from a variety of 
research settings as well as controlled studies of various approaches to accelerometer orientation and protocols are needed to determine the optimal approach for obtaining high adherence and low levels of loss.

\section{Acknowledgments}

The authors appreciate the involvement of Dara Brown, MPH; Mia Taylor, BS; Jorge Banda, MS; Mary Ellen Suitt, MPH; Meghan Baruth, PhD; Sherretta Thomas, MS; and Brent Hutto, MSPH. The project described was supported by Award Number R01DK074666 from the National Institute of Diabetes and Digestive and Kidney Diseases. The content is solely the responsibility of the authors and does not necessarily represent the official views of the National Institute of Diabetes and Digestive and Kidney Diseases or the National Institutes of Health.

\section{References}

1. Trost SG, McIver KL, Pate RR. Conducting accelerometerbased activity assessments in field-based research. Med Sci Sports Exerc. 2005;37:S531-S543.

2. Hawkins MS, Storti KL, Richardson CR, et al. Objectively measured physical activity of USA adults by sex, age, and racial/ethnic groups: a cross-sectional study. Int J Behav Nutr Phys Act. 2009;6:31. Available from http://ijbnpa. org/content/6/1/31. Accessed January 26, 2010.

3. Troiano RP, Berrigan D, Dodd KW, Masse LC, Tilert T, McDowell M. Physical activity in the United States measured by accelerometer. Med Sci Sports Exerc. 2008;40:181-188.

4. Sharp LK, Fitzgibbon ML, Schiffer L. Recruitment of obese black women into a physical activity and nutrition intervention trial. J Phys Act Health. 2008;5:870-881.

5. Escobar-Chaves SL, Tortolero SR, Masse LC, Watson $\mathrm{KB}$, Fulton JE. Recruiting and retaining minority women: findings from the Women on the Move study. Ethn Dis. 2002;12:242-251.

6. Staffileno BA, Coke LA. Recruiting and retaining young, sedentary, hypertension-prone African American women in a physical activity intervention study. J Cardiovasc Nurs. 2006;21:208-216.

7. Roberson NL. Clinical trial participation. Viewpoints from racial/ethnic groups. Cancer. 1994;74:2687-2691.

8. Mouton CP, Harris S, Rovi S, Solorzano P, Johnson MS. Barriers to black women's participation in cancer clinical trials. J Natl Med Assoc. 1997;89:721-727.
9. Ward DS, Evenson KR, Vaughn A, Rodgers AB, Troiano RP. Accelerometer use in physical activity: best practices and research recommendations. Med Sci Sports Exerc. 2005;37:S582-S588.

10. Zhu S, Heymsfield SB, Toyoshima H, Wang Z, Pietrobelli A, Heshka S. Race-ethnicity-specific waist circumference cutoffs for identifying cardiovascular disease risk factors. Am J Clin Nutr. 2005;81:409-415.

11. American College of Sports Medicine. ACSM's guidelines for exercise testing and prescription. 7th ed. Philedelphia: Lippincott Williams \& Wilkins; 2006.

12. Canadian Society for Exercise Physiology. PAR-Q and You (Physical Activity Readiness Questionnaire (PAR-Q) and Physical Activity Readiness Physical Examination, revised 2002). Available from http://www.csep.ca/CMFiles/publications/parq/par-q.pdf. Accessed July 20, 2009.

13. Parra-Medina D, Wilcox S, Wilson DK, Addy CL, Felton G, Poston MB. Heart Healthy and Ethnically Relevant (HHER) lifestyle trial for improving diet and physical activity in underserved African American women. Contemp Clin Trials. 2010;31(1):92-104.

14. Burroughs E, Peck LE, Sharpe PA, Granner ML, Bryant CA, Fields R. Using focus groups in the consumer research phase of a social marketing program to promote moderateintensity physical activity and walking trail use in Sumter County, South Carolina. Prev Chronic Dis [serial online]. 2006. Available from http://www.cdc.gov/pcd/issues/2006/ jan/05_0093.htm. Accessed August 10, 2009.

15. Matthews CE, Ainsworth BE, Thompson RW, Bassett DR, Jr. Sources of variance in daily physical activity levels as measured by an accelerometer. Med Sci Sports Exerc. 2002;34:1376-1381.

16. Masse LC, Fuemmeler BF, Anderson CB, et al. Accelerometer data reduction: a comparison of four reduction algorithms on select outcome variables. Med Sci Sports Exerc. 2005;37:S544-S554.

17. Freedson PS, Melanson E, Sirard J. Calibration of the Computer Science and Applications, Inc. accelerometer. Med Sci Sports Exerc. 1998;30:777-781.

18. Haskell WL, Lee IM, Pate RR, et al. Physical activity and public health: updated recommendation for adults from the American College of Sports Medicine and the American Heart Association. Circulation. 2007;116:1081-1093.

19. Herrmann S, Abraham T, Ainsworth B, Gilman M. Evaluating accelerometer cut-points to classify physical activity levels in overweight/obese middle-age-to-older women. Med Sci Sports Exerc. 2009;41:154.

20. Browning RC, Kram R. Energetic cost and preferred speed of walking in obese vs. normal weight women. Obes Res. 2005;13:891-899. 